\title{
Simulation of the all-vanadium redox flow battery with composite arched
}

\author{
channel \\ Jiangqi Zhou, Min Wang and Xin $\mathrm{Li}^{*}$ \\ School of Chemistry and Chemical Engineering, Beijing Institute of Technology, Beijing, 100081, \\ China \\ 1050072101@qq.com (J.Z.), 1142128092@qq.com (M.W.), klkxlx@163.com (X. L.)
}

Keywords: Vanadium flow battery, Mathematical model, Flow channel, Field synergy

Abstract. The all vanadium flow redox battery (VRB) has been widely studied and is one of the most compelling storage technologies. Here, A new channel structure, that is, a composited arched flow channel, is presented and compared with rectangular, serpentine channel. Numerical simulations are used to study the effects of channel structures on the concentration field, the temperature field, the pressure field and the charge-discharge curves in batteries. The results are analyzed based on the field synergy principle, which indicate that the composited arched channel has the advantages of homogeneous flow and low pumping costs and is more beneficial to battery performance.

\section{Introduction}

Since the 20th century, the energy crisis has become a serious impediment to sustainable development. play an important role in rapid economic development. The most compelling of such rechargeable batteries is the all-vanadium redox battery (VRB), which has important applications in many fields $[1,2]$. With the rapid development of VRB, mathematical model studies on VRB cell have also been conducted [3, 4]. In this paper, various flow channel configurations for a VRB are developed in this paper. The configurations include rectangular, serpentine, and composite arched channels. The principle of field synergy is used to analyze the results microscopically.

\section{Model assumption}

\section{Geometry model}

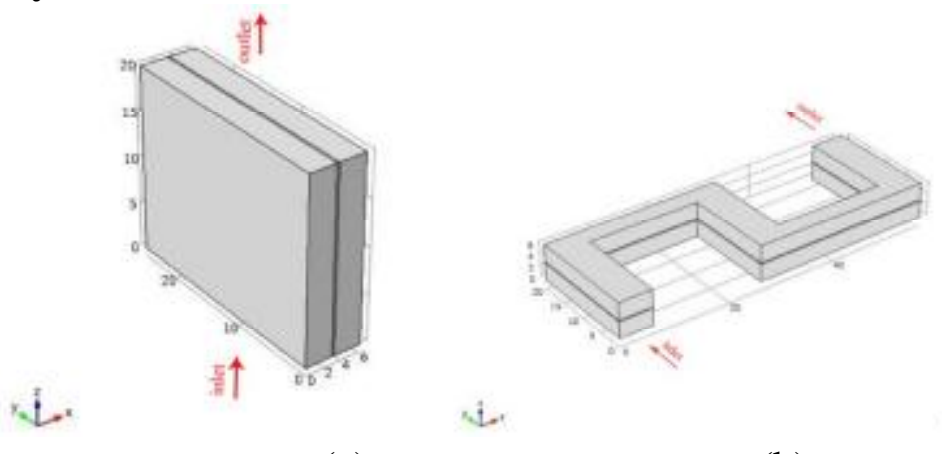

(a)

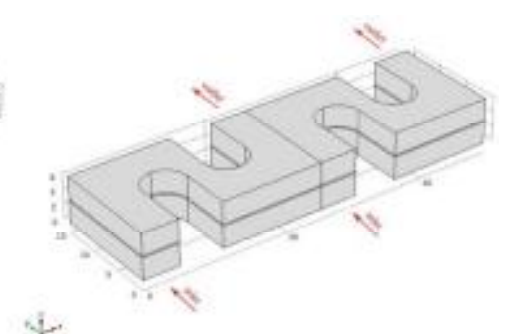

(c)

Fig. 1 Geometry models of VRB with different flow channels: (a) rectangular, (b) serpentine, and (c) composite arched channel structure

This work first examines the cell membrane-electrode assemblies (MEA) of a VRB to simplify the mathematical model. Sulfuric acid solutions containing vanadium oxide ions and vanadium ions 
are used as the positive and negative electrolyte, respectively, and are circulated to the battery by pumping. Fig. 1 shows three geometric models with different flow channel configurations: (a) rectangular, (b) serpentine, and (c) composite arched. Along x direction in (a) or the z direction in (b) and (c), the various regions are as follows (in $\mathrm{mm})$ : negative electrode $(0 \leq \mathrm{x}($ or $\mathrm{z})<3$ ); membrane $(3 \leq \mathrm{x}($ or $\mathrm{z})<3.25)$; positive electrode $(3.25 \leq \mathrm{x}($ or $\mathrm{z}) \leq 6.25)$. The composite arched channel is composed of two single arched channels, and the electrolyte flow rate in each channel is equal to half of the total flow rate. The inlets and outlets of these models are shown in Fig. 1.

\section{Equations}

The following assumptions have been made: (i) The fluid flow is treated as incompressible; (ii) The physical properties of the electrode, the electrolyte and the membrane are isotropic; (iii) Side reactions are not considered. Based on these assumptions, the governing equations are as follows:

Continuity: $\nabla \cdot \vec{v}=0$

Momentum conservation: $\frac{\mu}{K} \vec{v}=-\nabla P$ (electrode)

$$
\frac{-K_{w}}{\mu_{w}} \nabla^{2} P=0 \text { (membrane) }
$$

Among above, $\vec{v}$ is the velocity of the electrolyte flow, $\mathrm{m} / \mathrm{s}, \mathrm{K}$ is the permeability, $\mathrm{m}^{2}, \mathrm{P}$ is the pressure, Pa.

\section{Boundary conditions}

The all models were solved by using the commercial package COMSOL Multiphysics ${ }^{\circledR}$ with an instantaneous iterative solver. The models were coupled with the momentum equation, mass equation, energy equation and global ODEs and DAEs options. The model was divided by a triangulation method, and the relative error was set to $1 * 10^{-6}$. The flow rate of electrolyte is $0.5 * 10^{-6}$ $\mathrm{m}^{3} / \mathrm{s}$, pressure of outlets is $0 \mathrm{pa}$, surface area of electrode is $5^{*} 10^{-4} \mathrm{~m}^{2}$, volume of electrolyte is $3 * 10^{-5} \mathrm{~m}^{3}$.

\section{Results and discussion}

\section{Charge-discharge curves}

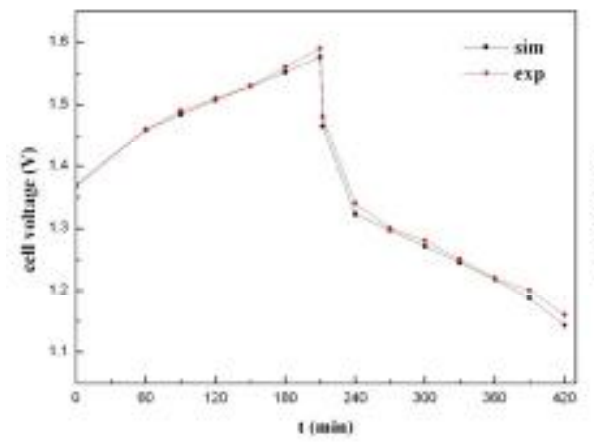

(a)

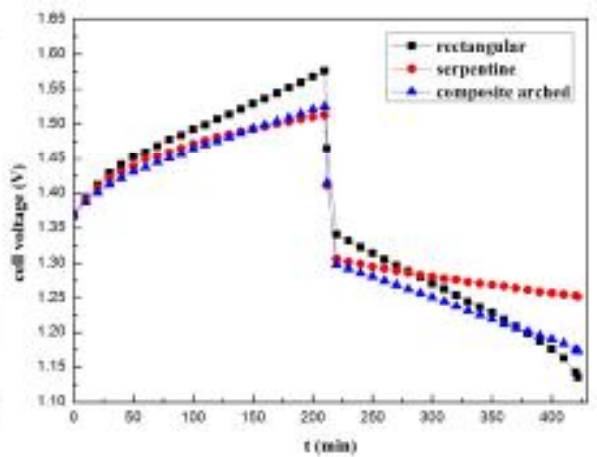

(b)

Fig. 2 A comparison of (a) simulated and experimental voltage values in a rectangular channel, (b) charge-discharge curves in different channels

Fig. 2a shows a comparison between the simulation and experiment values at different applied current densities in a rectangular flow channel. $0<\mathrm{t}<210 \mathrm{~min}$ corresponds to a charging process, 212 min $<\mathrm{t}<422$ min represents a discharging process, and in each case the simulation included a 2 -min rest period with zero current before discharge. The maximum error is $1.5 \%$, demonstrating that the model captures the trends extremely well. The sources for the errors may be the assumptions that all pores in electrode are considered to be of the same size, and the physical 
properties are isotropic. In reality, the sizes of the pores are not quite the same, and the properties are actually anisotropic.

Fig. $2 b$ shows the influence of the flow channel structure on the charge-discharge curve. The results indicate that the polarization in the rectangular channel is the largest, while the battery performance in the composite arched channel is the most stable. Compared with the others, although the flow rate is low in the composite arched channel, the electrolyte flow is regular, the resistance is small, and the cell works stably. Moreover, the calculations show that the energy efficiency is $81.38 \%, 83.8 \%$ and $80.7 \%$ in the composite arched, serpentine and rectangular channels, respectively.

\section{Concentration fields}

For this paper, the concentration distribution of $\mathrm{V}^{3+}$ at the negative electrode at a particular time $(\mathrm{t}=10 \mathrm{~min})$ during charging was selected as an example. For discharge, the trends are reversed.

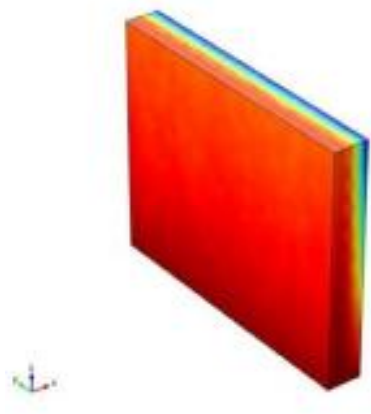

(a)

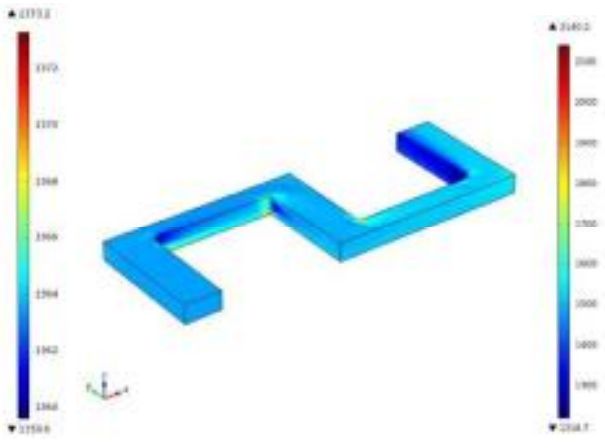

(b)

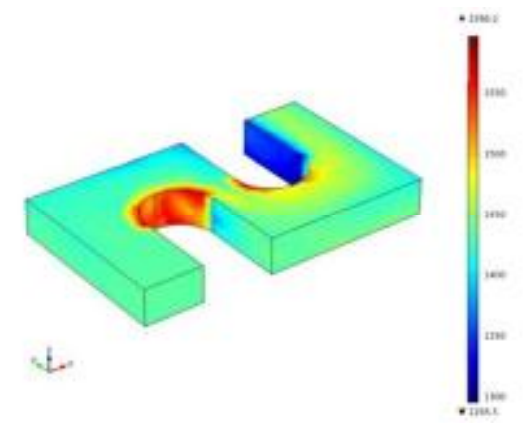

(c)

Fig. 3 The concentration field in different flow channels

Fig. 3 (a), (b) and (c) show the concentration field of $\mathrm{V}^{3+}$ in the rectangular, serpentine, and composite arched channel models, respectively. The concentration decreases as electrolyte flows from the inlet to the outlet, and farther from the electrode-membrane interface, the concentration gradient is steeper. As the reaction takes place at the electrode surface, $\mathrm{V}^{3+}$ as a reactant is consumed. This consumption produces a concentration gradient and promotes a liquid transfer process.

Notably, at the corner of the ridge in (b) and (c), the concentration varies sharply, and the concentration gradient in (b) is relatively steeper. When the electrolyte flows through, it encounters greater resistance than does the internal flows. Compared with the serpentine channel, the shape of the ridge in composite arched channel is an arc, and the electrolyte flows homogeneously.

\section{Temperature fields}

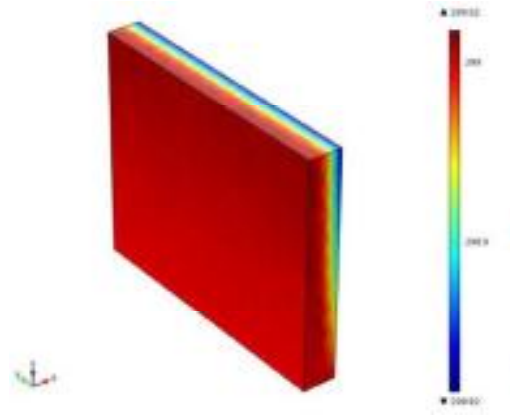

(a)

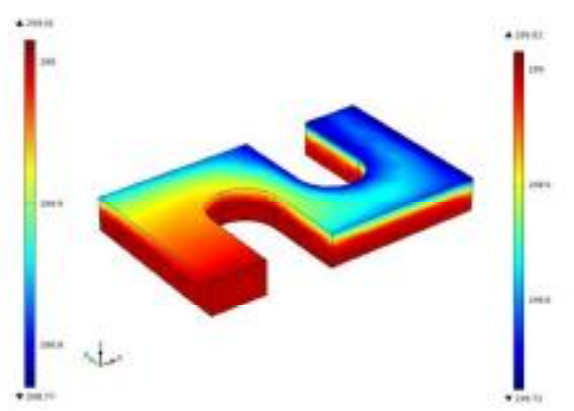

(c)

Fig. 4 Temperature field in different flow channels

The temperature variations at $\mathrm{t}=10 \mathrm{~min}$ during charging were selected examples. For discharge the trends are reversed. Fig. 4 shows the temperature fields in the (a) rectangular, (b) serpentine, and 
(c) composite arched models. In addition, the temperature drops gently through each system, but the gradient varies sharply near the electrode surface. It is notable that the temperature is more stable in (c) than the others. During charging, the cell absorbs heat energy to make electrical energy convert to chemical energy for storage. Therefore, the influence of the temperature field on the battery is less significant as the time goes on.

\section{Pressure field}

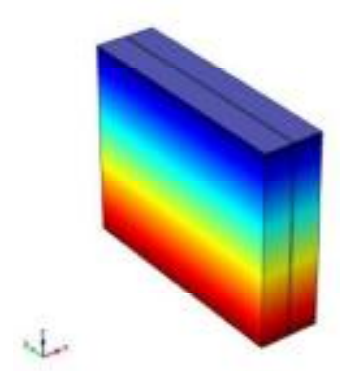

(a)
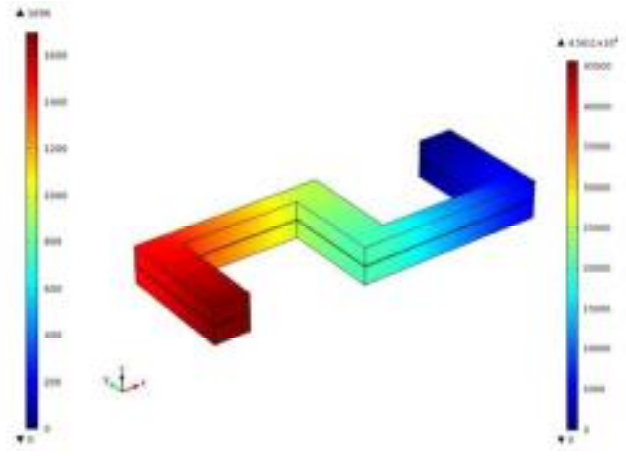

(b)

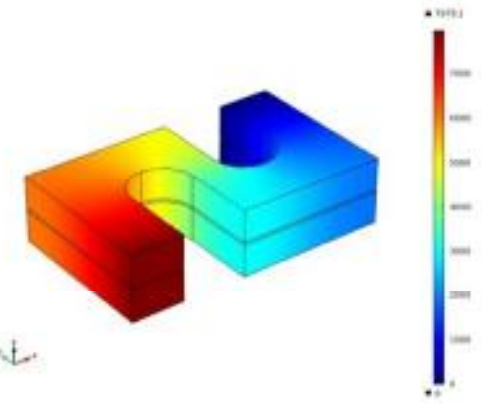

(c)

Fig. 5 Pressure field in different flow channels

The steady-state form of Darcy's law is applied for momentum conservation. Thus, the pressure distribution is constant in time. Fig. 5 shows the pressure field in the different flow channel models of (a) rectangular, (b) serpentine, and (c) composite arched. As is shown above, the electrolyte pressure decreases gradually from the inlets to outlets. Comparing the figures above, the order of pressure drop is the following: rectangular < composite arch < serpentine, with values of $1700 \mathrm{~Pa}$, $46 \mathrm{kPa}$ and $16 \mathrm{kPa}$, respectively. During operation, pumping provide an electrolyte static pressure, which is converted into kinetic energy to make the electrolyte flow between the storage tank and the battery. Therefore, the higher the pressure drop, the greater the energy consumption of the pump.

According to the comprehensive analysis above, the composite arched channel is more stable and is more suitable for the long-term cycle of a VRB than the other configurations because the composite arched channel reduces the polarization and side reactions that can occur at higher current densities.

\section{Field synergy analysis}

The interaction between different fields to strengthen the process is known as the "field synergy ". That is, the intensity of one process not only depends on the magnitude of the field vector but also the angles at which the different fields interact with each other [5].

MEA is a process in which heat transfer, mass transfer and electrochemical reactions are coupled. Factors affecting mass transfer primarily include the chemical potential gradient (concentration gradient), the temperature gradient (Soret effect), and the velocity field. The angle of the chemical potential gradient between all species is maintained near $0^{\circ}$; therefore, the synergistic relationship remains the same and will not be further considered here. 


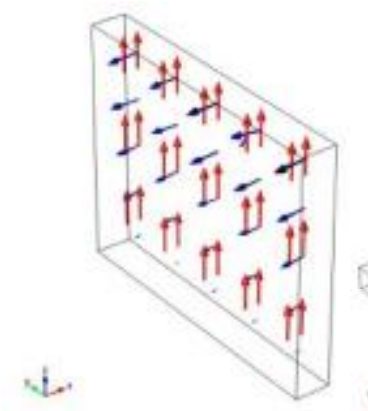

(a)

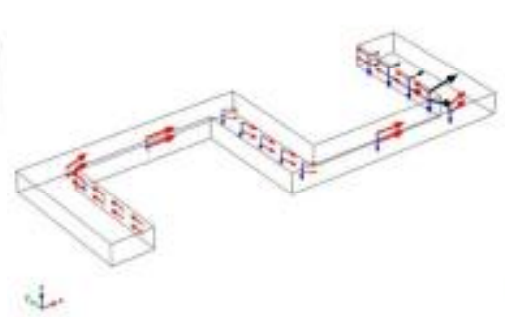

(b)

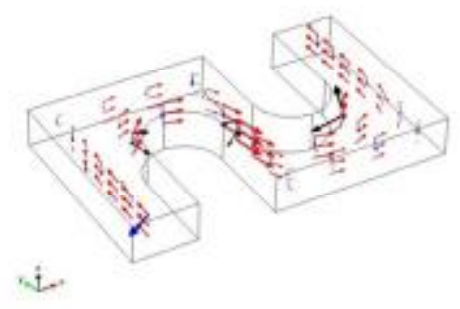

(c)

Fig. 6 Synergic relationship in different flow channels.

Fig. 6a shows the synergic relationship in a rectangular channel. As shown above, the red, blue and black arrows represent the velocity field, the concentration gradient field of $\mathrm{V}^{3+}$ at the negative electrode, and the temperature gradient field, respectively. The arrow directions represent the directions of the fields and the lengths of the arrows represent the intensities of the fields. The angle of concentration gradient and the velocity field is close to $90^{\circ}$; therefore, the synergistic relationship is smaller; meanwhile, the angles of the concentration gradient and temperature gradient are nearly $0^{\circ}$, meaning that the synergistic relationship is larger. That is to say, a more effective way to improve mass transfer is to change the synergy angles of the concentration gradients and the velocity fields. In terms of heat transfer, the temperature gradient and velocity fields become vertical, which weakens the heat transfer effect and is good for battery performance.

Fig. $6 \mathrm{~b}$ shows the synergic relationship in a serpentine channel. In terms of heat transfer, the angle of the temperature gradient and the velocity field is close to $90^{\circ}$, which is beneficial to battery performance. Compared with a rectangular channel, there are many differences in mass transfer. On one hand, the angles of the velocity field and concentration gradient field are larger than $90^{\circ}$, which could strengthen the mass transfer. On the other hand, the Soret effect weakens the internal mass transfer and is adverse to the battery performance. Due to the overall temperature changes being smaller, the influence of the Soret effect could be ignored in this case.

Fig. 6c shows the synergic relationship in a composite arched channel. The synergic relationship is similar to that observed in a serpentine channel. However, compared with Fig. 6b, the electrolyte flows more homogeneously, and the synergic angles of the concentrate fields and velocity fields are larger, both of which strengthen the mass transfer.

\section{Conclusion}

Membrane-electrode models of a VRB with rectangular, serpentine and composite arched flow channel configurations are developed in this paper. The comprehensive analysis of economic efficiency and operating performance analysis demonstrate that the composite arched flow channel has the advantages of a small flow resistance, a short flow path and is more suitable for the overall performance of a VRB. Moreover, the field synergy principle analysis indicates that the use of a composite arched channel will effectively change the synergy angle of the field in the cell and largely strengthen the mass transfer process. In short, the composite arched channel configuration provides a greater improvement in battery performance compared to the other two configurations.

\section{Acknowledgements}

This work was supported by Beijing Institute of Technology scientific cooperation project (No. 3190012351701) and the National Nature Science Foundation of China (No.21111120074). 


\section{References}

1. G. Kear., A. A. Shah., F. C. Walsh: Int, J. Energy Res 36, (2012), 1105-1120.

2. Minke, C., Turek, T: J. Power Sources 376, (2018), 66-81.

3. C.K. Jia., J.G. Liu., C.W. Yan: J. Power Sources 203, (2012), 190-194.

4. Chu, D., Li, X., et.al: Electrochimica Acta 2016, (190), 434-445.

5. Z.Y. Guo., D.Y. Li., B.X. Wang: Int. J. Heat Mass Transfer 41, (1998), 2221-2225. 